\title{
EL TRABAJO COEDUCATIVO Y LA IGUALDAD DE GÉNERO DESDE LA FORMACIÓN INICIAL EN EDUCACIÓN FÍSICA
}

\author{
David Hortigüela Alcalá \\ Alejandra Hernando Garijo \\ Universidad de Burgos
}

RESUMEN: El objetivo del estudio es comprobar en qué medida un enfoque participativo en la formación inicial del profesorado (FIP) de Educación Física (EF) contribuye a que el alumnado valore positivamente el uso de estrategias vinculadas con la educación y el trabajo de género. Esto se contrasta con las valoraciones que realiza el personal experto acerca de su empleo en la situación educativa actual. La investigación se realizó en la Facultad de Educación de la Universidad de Burgos. Participaron 51 alumnos/as de Grado de Educación Primaria y siete docentes universitarios, de diversas universidades, expertos en el tratamiento de la EF. Se ha utilizado una metodología cualitativa, siendo los grupos de discusión con alumnado y las entrevistas a docentes los instrumentos de recolección de datos. Los resultados muestran cómo tras vivenciar los contenidos prácticos de la asignatura, los/as futuros/as maestros/ as consideran haber descubierto el área como una herramienta idónea para el trabajo coeducativo y de género. Las personas expertas manifiestan que se necesita una reorientación en la EF escolar hacia las relaciones interpersonales y la cooperación. Se concluye acerca de la relevancia que tiene seguir conexionado la práctica escolar con la FIP respecto a la coeducación y el trabajo de género.

PALABRAS CLAVE: Coeducación, igualdad de género, educación física, formación inicial del profesorado.

\section{COEDUCATIONAL WORK AND GENDER EQUALITY SINCE INITIAL TRAINING IN PHYSICAL EDUCATION}

\footnotetext{
ABSTRACT: The objective of the study is to verify how a participatory approach in initial teacher training (ITT) of Physical Education (PE) helps students to value positively the use of strategies related to education and gender work. This is contrasted with the assessments that experts make about their
} 
use in the current educational situation. The research was carried out in the Faculty of Education of the University of Burgos. Fifty-one students participated in the Primary Education Degree and seven university teachers, from different universities, experts in the treatment of PE. A qualitative methodology has been used, being the groups of discussion with students and the interviews to teachers the instruments of data collection. The results show how after experiencing the practical contents of the subject, future teachers consider having discovered the area as an ideal tool for coeducational and gender work. Experts say that a reorientation in PE school is necessary for interpersonal relationships and cooperation. It concludes about the relevance of linking school practice with the ITT regarding co-education and gender work.

KEYWORDS: Co-education, gender equality, physical education, initial teacher training.

Recibido: 23/03/2017

Aceptado: 13/07/2017

Correspondencia: David Hortigüela Alcalá, Universidad de Burgos, Facultad de Educación., Departamento de Didácticas específicas, C/ Villadiego, 1, 09001 Burgos. Email: dhortiguela@ubu.es.

\section{INTRODUCCIÓN}

Son varias las investigaciones que indican que la EF escolar puede ser una asignatura idónea para generar valores positivos en el alumnado en cuanto a ła igualdad, respeto, tolerancia y aceptación de las limitaciones y posibilidades propias y de los demás independientemente del sexo y/o raza (Hortigüela, Fernández-Río y Pérez-Pueyo, 2016a; Hortigüela, Fernández-Río y Pérez-Pueyo, 2016b). Sin embargo, también puede convertirse en una materia que fomente la individualización, la frustración y la falta de compañerismo y aceptación si no se utilizan metodologías reflexivas de carácter abierto y participativo (Dervent, 2015). Es fundamental por lo tanto establecer prácticas pedagógicas que hagan que los/as estudiantes se sientan parte de lo que están aprendiendo y que así no sean meros receptores de información.

En este sentido, la manera en la que se enfoque la asignatura y el rol que adquiera el profesorado en el aula y con el alumnado son dos factores fundamentales para la generación de conductas y valores positivos en los/as estudiantes (Bozkurt y Tel, 2016). Variables como la actitud hacia las tareas y hacia los demás, conductas de actuación positivas y de aceptación en las prácticas deportivas, adquisición de pautas de respeto y tolerancia hacia sus iguales y el desarrollo de una autonomía para la superación de retos y obstáculos, han demostrado ser adquiridas cuando el enfoque pedagógico de la materia no se centra únicamente en el rendimiento físico y deportivo (Finco et al., 2015).

Es por ello trascendental que se establezca una conexión real y directa entre lo que acontece en la materia de EF escolar y aquello que se enseña en la FIP. Si somos 
conscientes de la gran cantidad de cambios existentes actualmente a nivel social, de intereses del alumnado y de sus formas de relacionarse, es en la formación del profesorado desde donde debemos dirigir los esfuerzos para generar revoluciones y cambios de dinámicas que aborden los problemas actuales (Parker, Bush y YendolHoppey, 2016). Para ello, y tras haberse demostrado reiteradamente que el trabajo de contenidos teóricos y prácticos aislados no genera el conocimiento y las competencias deseadas, es preciso cambiar estructuralmente la docencia y dirigirla hacia una integración con el ámbito emocional y de género (Chick, 2014). Esto permite extraer todo el potencial motor y corporal de la EF e integrarlo en propuestas coeducativas con plena significación personal y relacional. Si un/a futuro/a docente recibe en su formación inicial una idea de trabajo corporal desde una perspectiva de igualdad y de autoconocimiento personal, es mucho más probable que la aplique en un futuro.

Bajo estos postulados se intentan transformar las realidades sociales, implicando en la medida de lo posible a la comunidad educativa. Si todavía hoy en día seguimos en la búsqueda de una sociedad más tolerante, empática y respetuosa con los demás, parece irrevocable que se reme en la misma dirección, entendiendo la escuela como ese lugar de pleno desarrollo de las capacidades afectivas del alumnado que contribuya a una mayor justicia social (Mills, McGregor, Baroutsis, Te Riele y Hayes, 2016). Para ello, desde la EF, sería deseable realizar trabajos directamente relacionados con las familias mediante propuestas temporalizadas, planificadas y consensuadas que permitan comprobar los efectos pretendidos.

Aunque ya hace años éramos conscientes de los problemas que acontecían en la escuela acerca del acoso, discriminación, faltas de respeto e intolerancia hacia los/as compañeros/as, es en los últimos tiempos cuando ha estallado verdaderamente esta preocupación por casos de gravedad que han tenido repercusión en los medios de comunicación. Parece que ahora existe una mayor conciencia a la hora de no mirar para otro lado cuando suceden estas conductas, independientemente de si se han producido o no específicamente en los centros educativos. Además, el "boom" del uso de las redes sociales y de diversos canales de comunicación, cada vez desde edades más tempranas, ha provocado que sea más sencillo efectuar, fuera del horario escolar, el abuso y el acoso sobre alumnos/as emocionalmente más débiles (Khasnabis y Upton, 2013). Esto se relaciona directamente con la educación de género, siendo varios los estudios que indican cómo muchas alumnas presentan un rol de sumisión y aceptación del poder del varón, por ejemplo en la toma de decisiones del día a día (Kingdon, Serbin y Stack, 2017). También es relativamente habitual, a pesar del elevado número de casos de violencia de género que acontecen en nuestro país, que las adolescentes justifiquen acciones violentas de sus parejas por ataques de celos, mirar a otros chicos o vestir con un determinado tipo ropa. Este tipo de actuaciones, que la sociedad en su inmensa mayoría condena, no son en muchos casos correspondidas desde los colegios e institutos con protocolos de actuación para concienciar, prevenir, detectar y actuar contundentemente cuando suceden. Tales acciones, nada pedagógicas, pueden verse transmitidas en EF a través del currículo oculto, cuando el/la docente muestra subconscientemente ejemplos y/o comentarios que debilitan la visión del sexo femenino en el ámbito físico y motriz. Ante este panorama, la EF, desde un enfoque alternativo y a través del trabajo conjunto de otras áreas, puede convertirse en una de las piedras angulares del cambio, debido fundamentalmente 
al plano vivencial y experimental sobre la que se configura (Temel y Güllü, 2016). Por lo tanto, y con el fin de establecer un análisis de diagnóstico que pueda generar cambios en los/as futuros/as maestros/as de la materia, consideramos imprescindible atender al impacto que tienen nuestras prácticas educativas en la percepción del alumnado en la FIP. Esto, complementado con la valoración de expertos en la temática, puede dar una información verdaderamente valiosa. Así, el aporte relevante de este estudio es demostrar en qué medida el empleo de metodologías de carácter cooperativo y relacional en EF influyen en la percepción positiva de futuros/as maestros y maestras para utilizar estrategias de trabajo coeducativo en su futuro desempeño profesional. Además, contrastar sus valoraciones con la de personas expertas en este ámbito ofrece resultados que contribuyen significativamente a la literatura existente sobre la temática. De este modo, los objetivos de estudio son: a) Comprobar la incidencia que tiene el enfoque metodológico de la enseñanza de la EF en la valoración y la adquisición de estrategias prácticas para el trabajo de la coeducación y la igualdad de género en futuros/as maestros y maestras; b) Analizar la valoración de personas expertas en el ámbito de la EF (profesorado e investigadores/as) sobre el rol que desempeña actualmente el trabajo de la coeducación y la igualdad de género en la escuela.

\section{Material y métodos}

\section{Participantes}

Han participado 51 alumnos/as (29 mujeres y 22 hombres) matriculados en la asignatura de Educación Física y su Didáctica de segundo curso (segundo semestre) del Grado de Maestro de Educación Primaria en la Facultad de Educación de la Universidad de Burgos. Tienen una media de edad de $20.57 \pm 2.38$ años. También participaron siete docentes universitarios (cuatro mujeres y tres hombres) expertos/as en el tratamiento de la EF desde una visión pedagógica e integradora. Estos investigadores/as, con multitud de publicaciones científicas en este ámbito, pertenecen a las Universidades de Burgos (2), de Valladolid (2), de Santander (1), Autónoma de Madrid (1), y de Granada (1). Todos y todas forman parte del Área de Didáctica de Expresión Corporal y de Educación Física y Deportiva. Su media de edad es de $45.87 \pm 8.13$ años y $11.76 \pm 6.35$ años de experiencia en el ámbito universitario. Uno tiene la categoría de Catedrático de Universidad, dos de ellas de Titular de Universidad, una de Contratado Doctor, dos de Ayudante Doctor y uno Asociado 6+6.

Todos/as los/as participantes de la investigación fueron seleccionados/as por criterios de accesibilidad, representatividad y aporte al objeto de estudio. Los alumnos y alumnas del Grado en Primaria por formar parte de la asignatura, y el personal experto, referencias a nivel nacional/internacional de la materia, por su predisposición positiva hacia la investigación.

\section{Instrumentos}

Se llevó a cabo un grupo de discusión con el alumnado participante al finalizar la asignatura. Esta técnica de análisis en la investigación cualitativa, y más en el ámbito educativo, favorece el intercambio de ideas, la profundización en la temá- 
tica y el contraste multidimensional de percepciones de los/as estudiantes (Choi y Protivnak, 2016). Además, la presencialidad y el cuestionamiento de ideas vinculadas directamente a los aspectos prácticos y vivenciales experimentados por el/la discente a lo largo de sus estudios garantiza una mayor riqueza e interacción en el debate (Rosen, Scott y DeOrnellas, 2017). Si bien parte de la literatura determina que un grupo de discusión ha de tener un número comprendido entre 7 y 12 participantes, existen diversas experiencias de éxito que han empleado grupos focales estructurados de mayor número con delimitación de responsables bajo modelos de debate (Varga-Atkins, Mclsaac y Willis, 2015). Esta fue la técnica empleada. Los/ as 51 estudiantes se dividieron en 6 grupos proporcionados que debatían internamente durante tres-cuatro minutos para emitir posteriormente sus respuestas a la pregunta planteada. A partir de aquí, y antes de pasar a la siguiente cuestión, se establecía la discusión intergrupos a través del rol de moderador o moderadora adquirido por los/as investigadores. Por otro lado, con el personal experto se realizaron entrevistas individuales semiestructuradas. Cuatro de ellas fueron presenciales y las otras tres on-line. El dotar de un carácter individual a las técnicas de recogida de datos cualitativos, más si cabe cuando las personas son expertas, conlleva una profundización mayor en la información abordada (Costigan, 2009). Tanto el grupo de discusión como las 4 entrevistas presenciales fueron grabados en vídeo. Las tres entrevistas on-line a las personas expertas también fueron registradas por el programa Hangout. Todo ello permite una mayor facilidad en la posterior transcripción de los datos.

Tanto en los grupos de discusión como en las entrevistas se emplea un guion estructurado con una serie de cuestiones vinculadas al objeto de estudio. En el grupo de discusión las preguntas se dirigen a conocer la percepción del estudiante sobre la experiencia obtenida en EF respecto al trabajo de coeducación e igualdad de género. Al personal experto se les preguntó sobre el enfoque, rol e importancia que tiene la EF escolar y la formación de maestros y maestras en estos aspectos.

En la tabla 1 se reflejan las cuestiones empleadas con el grupo de los alumnos/as.

Tabla 1. Guión básico utilizado para el grupo de discusión con los alumnos

1. ¿Consideráis que el trabajo de coeducación y de género ha de abordarse implícitamente en las escuelas? ¿Por qué esto no se hace todavía?

2. ¿Estáis recibiendo formación al respecto en la universidad cómo futuros maestros?

3. ¿Pensáis que desde la EF se puede contribuir positivamente al trabajo coeducativo y de género? ¿cómo?

4. ¿Ha cambiado vuestra percepción una vez vivenciada la experiencia en la asignatura de didáctica de EF? ¿en qué?

5. ¿Cuál creéis que debería de ser el enfoque que se diera a la EF escolar?

6. ¿Por dónde se debería de empezar a trabajar para poder aplicar un modelo integral de EF inclusiva? 
En la tabla 2 se reflejan las cuestiones empleadas con las entrevistas a expertos/as.

Tabla 2. Guión básico utilizado para las entrevistas con los profesores expertos

1. ¿Se trabaja lo suficiente en la formación inicial de maestros el tema de coeducación y de género? ¿Por qué?

2. ¿Se trabaja lo suficiente en los colegios el tema de coeducación y de género? ¿Por qué?

3. ¿Está aportando actualmente la EF escolar a este ámbito? ¿Por qué?

4. ¿Qué cosas habría que cambiar en la EF escolar para que la coeducación y el trabajo de género se encuentren verdaderamente implícitas en la práctica?

5. ¿Está el profesorado preparado para estos cambios? ¿Por qué?

6. ¿Qué tipo de propuestas son las más favorecedoras para relacionar EF con trabajo inclusivo?

\section{Diseño y procedimiento}

Los/as 51 estudiantes participaron en la experiencia desarrollada en la asignatura de EF y su didáctica de $2^{\circ}$ curso del Grado en Educación Primaria. Desde el comienzo se le comentó al alumnado el propósito de la experiencia. La asignatura se estructuró desde una perspectiva formativa, rompiendo el carácter tradicional que configura la EF bajo parámetros exclusivos de rendimiento motriz y deportivo. Para ello, el movimiento se utilizó como una herramienta para el fomento de relaciones interpersonales positivas, el desarrollo de valores y la obtención de experiencias emocionales significativas. De este modo, toda la práctica giró a partir del planteamiento de supuestos reales en centros educativos, generando dilemas morales que ponían a los/as futuros/as docentes en situaciones constantes de reflexión. Para resolverlos, grupalmente, tenían que plantear estrategias metodológicas desde el área de EF que dieran respuesta a cada caso, siempre a través de un enfoque de perspectiva de logro, tolerancia, aceptación de las características del grupo clase e inclusión. A colación de este último término, es preciso aclarar su significado, ya que el mismo se utiliza en el presente estudio como un enfoque de enseñanza que responde positivamente a la diversidad de las personas y a las diferencias individuales, entendiendo que la diversidad es una oportunidad para el enriquecimiento de la sociedad y de la educación.

Para todo ello, la reflexión era necesaria y constante en cada una de las tareas, ya que el/la futuro/a docente de EF debía utilizar el cuerpo y el movimiento como elemento de integración, comprensión y aceptación entre iguales. Este trabajo, regulado a través de la aplicación de un sistema de evaluación formativa y compartida, tuvo una duración de 14 semanas, tiempo que duró la asignatura. Los alumnos y las alumnas formaban parte de su propia evaluación, regulando su proceso de aprendizaje y estableciendo juicios de valor a sus compañeros y compañeras durante la asignatura, con el fin de ser críticos/as y más conscientes del aprendizaje generado. Todas las tareas llevadas a cabo en la asignatura tuvieron un carácter grupal, desarrollando 
sesiones prácticas y analizando casos reales en EF que podrían generar desigualdad de género. Para ello, existía una retroalimentación entre profesor y cada uno de los grupos, así como entre grupos, para mejorar las argumentaciones de las soluciones encontradas con el fin de paliar esa discriminación. Cada grupo registraba su trabajo semanal en un diario, utilizando así las técnicas narrativas y el pensamiento reflexivo para empatizar y sensibilizarse con la coeducación e igualdad de género en EF. El compromiso de cada uno de los grupos era elevado, ya que tenían que registrar cuáles eran la percepciones de sus integrantes para al finalizar la asignatura hacer un reparto de notas (Pérez-Pueyo, Hortigüela y Hernando, 2014). Los contenidos abordados en las clases prácticas, desarrolladas en el polideportivo y en el aula de expresión corporal, se estructuraron a través del aprendizaje cooperativo, con la premisa fundamental de la importancia que tiene la responsabilidad y el logro del conjunto por encima de las individualidades. Así, se plantearon diferentes retos motrices con diverso material como colchonetas, aros, balones..., en los que únicamente se podrían lograr satisfactoriamente si todos y todas pensaban como equipo. La tarea conllevaba una reflexión inicial para buscar la mejor forma de actuar. Esto les hacía ver que en un grupo, independientemente de sus características, todos y todas eran imprescindibles, Ilegando más lejos si juntamos nuestras capacidades. Paralelamente al desarrollo de cada taller práctico, los grupos anotaban en su diario de campo la manera en la que habían resuelto los conflictos generados y dónde podrían encontrarse desigualdades al desarrollar esta práctica en el aula de primaria. Este componente reflexivo asociado a la práctica, derivaba posteriormente en debates muy interesantes con aportaciones de todos los grupos.

Paralelamente al desarrollo de la asignatura se diseñó la investigación y se contactó con los investigadores/as expertos/as en la temática del estudio. Se les comentó los objetivos y se les preguntó si estaban dispuestos/as a colaborar. Todos/as mostraron un interés elevado, por lo que se fijaron las fechas tanto para las entrevistas presenciales como para las on-line. Finalizada la asignatura se realizó tanto el grupo de discusión como las entrevistas, registrando la información y procediendo a su análisis posterior. A partir de aquí se determinaron las categorías de análisis. Con el alumnado se realizó en el aula habitual en la que se impartía clase. Con los cuatro docentes presenciales en un seminario de la facultad. El grupo de discusión tuvo una duración de 90 minutos y cada una de las entrevistas alrededor de 45.

A todos/as los/as participantes se les aseguró la confidencialidad en las respuestas y el anonimato, garantizando que únicamente se utilizarían los resultados con fines científicos. También se les comentó que los resultados obtenidos eran abiertos, por lo que podían ser compartidos sin ningún problema.

\section{Análisis empleado}

Se utiliza una metodología cualitativa, profundizando y analizando las experiencias y vivencias de los sujetos participantes. Se pretende con ello extraer de primera mano toda la información referente a procesos educativos innovadores que generen cambios y aporten a situaciones sociales actuales y relevantes (Thorleif, 2007). Los datos obtenidos en este caso son complementarios, ya que provienen de futuros/ as maestros/as que han recibido la experiencia y de investigadores relevantes en el 
ámbito de la EF y la coeducación. Esta doble vía favorece la interrelación y el contraste de las argumentaciones establecidas en torno a la EF como favorecedora de la coeducación y género.

La información del grupo de discusión y de cada una de las entrevistas fue triangulada y saturada a partir de la generación de categorías y subcategorías derivadas de la codificación de los extractos de texto más significativos (Saldaña, 2009). Todos los resultados fueron analizados independientemente por los investigadores e investigadoras, contrastándolo después bajo los mismos criterios de análisis. A partir de la "Grounded Theory" (Strauss y Corbin, 2002) y de la teoría fundamentada de los datos, se relacionó la información obtenida en cada una de las preguntas con los datos causales que las generaban. Para ello se cruzaron los datos de las subcategorías que atendían a criterios comunes, fragmentando y articulando las ideas más representativas.

La asignación del texto a cada categoría se hizo de manera específica tras la lectura detallada de todo el volumen de datos. La codificación fue axial, abierta y selectiva. Se contabilizó la totalidad de los extractos de texto obtenidos en cada categoría, mostrando en resultados los más saturados y significativos.

\section{Generación de categorías}

Tras la recogida de información, el visionado de los vídeos y la trascripción de los datos, se procedió a la generación de las categorías de análisis. Para ello se atendió tanto a los parámetros de saturación de texto obtenidos como al mantenimiento de la linealidad con los objetivos del estudio. Para todo el análisis de resultados se empleó el programa de computación de datos WEFT QDA. Las categorías utilizadas fueron:

- Importancia del trabajo de la coeducación y de la igualdad de género: se analizan aspectos relacionados con la presencia que tienen estas variables actualmente en las escuelas, la formación que reciben futuros maestros y maestras, la manera en la que debería de ser abordada y las causas que justifican su implantación más manifiesta.

- Rol de la EF para el trabajo inclusivo en el aula: se registran los aspectos relacionados con la manera en la que desde la EF se puede trabajar la coeducación y la igualdad de género, las vivencias experimentadas por el alumnado al finalizar la asignatura, así como los aspectos relacionados con las características de la EF que pueden favorecer el trabajo igualitario y de aceptación a los demás.

- Enfoques y modelos pedagógicos aplicados a la EF para conseguir la tolerancia, igualdad y respeto en el aula: los aspectos que aquí se analizan se encuentran vinculados con los enfoques, técnicas y modelos pedagógicos con los que se debiera de aplicar la EF escolar desde una vertiente integradora. También se relaciona con la formación del profesorado.

Cada categoría se encuentra en linealidad con cada una de las cuestiones planteadas en el grupo de discusión con alumnos y alumnas, y las entrevistas al personal expertos, lo que favorece el ajuste entre la información recabada, la especificación 
de la misma y los objetivos del estudio. Esto permite una mayor exhaustividad y comprensión de los datos (Romano, Donovan, Chen y Nunamaker, 2014).

\section{Codificación de los extractos de texto}

Para la mejor interpretación de los datos se han utilizado diferentes acrónimos, asignado uno al grupo de discusión y uno específico a cada investigador/a experto/a. Para el grupo de discusión con futuros/as maestros/as se empleó (GDM), y para cada una de las entrevistas de los expertos/as se utilizó (EE1), (EE2), (EE3), (EE4), (EE5), (EE6), (EE7).

\section{Resultados}

A partir de los patrones cruzados de contenido se muestran, en cada una de las tres categorías del estudio, los extractos de texto más significativos y coincidentes. Además se contabilizan los mismos.

\section{Importancia del trabajo de la coeducación y de la igualdad de género (236 extractos de texto)}

Se observa cómo el alumnado valora muy positivamente el tratamiento que se ha hecho en la asignatura de EF, destacando que a lo largo de su formación se ha carecido de este tipo de enfoques a las asignaturas:

Me ha cambiado mucho la percepción que tenía de la asignatura de EF, sobre todo comparando con cuando la cursé en la ESO [...]. Afrontarla desde una metodología cooperativa hace que la inclusión sea mucho mayor y se tengan en cuenta las características de todos y de todas [...]. Me ha impactado mucho comprobar cómo a partir del movimiento se pueden tener relaciones personales muy buenas, ya que no importa si estás con un chico o con una chica, lo que importa es que todos consigamos el reto. La verdad es que me ha encantado, creo que necesitamos más asignaturas como estas donde el trabajo de grupo y la coeduación verdaderamente se viva y no se aborde de manera tan teórica (GDM).

Por otro lado, los expertos creen, que si bien el enfoque actual de la EF está cambiando en los últimos años, todavía sigue primando las propuestas puramente basadas en el rendimiento deportivo y no centradas en aspectos relacionales e integradores.

En los últimos 10 años la forma en la que se entiende y aborda la EF en la escuela ha cambiado, aunque todavía queda mucho por hacer [...]. (EE3). Los enfoques fisiológicos o plenamente lúdicos todavía son muy extendidos y por desgracia el ámbito social, emocional y relacional queda en un segundo plano (EE5). Ya está demostrado que la EF es una materia idónea para la prevención de conflictos en educación ¿esto no es lo suficientemente importante como para que sigamos haciendo lo mismo que hace 30 años? [...] (EE1). La asignatura ha de adaptarse a los problemas sociales actuales, por eso es fundamental que profesionales que desempeñarán en un futuro la materia de EF se les forme en esto. (EE7). De nada sirve que un niño o niña sea motrizmente muy bueno si luego es un déspota, intransigente e intolerante [...]. Ahí es donde está la verdadera fuerza de la EF. (EE2). 


\section{Rol de la EF para el trabajo inclusivo en el aula (221 extractos de texto)}

El alumnado afirma que la manera en la que se aborde la EF puede influir directamente tanto en la motivación de los/as estudiantes hacia la asignatura como en el clima de grupo que se genere, independientemente de las características de la clase o de sus diferencias personales y/o de género:

Con las actividades que hemos realizado en la asignatura te das cuenta de que todo el mundo puede conseguir las cosas que propone el profesor, pero para ello necesitas al grupo [...]. Todos y todas tenemos algo que aportar. Hemos trabajado mucho el currículo oculto y ves como implícitamente a veces se dan ejemplos en las clases totalmente machistas [...]. Así, las chicas poco a poco van asumiendo la superioridad de los hombres en muchas cosas. La EF puede aportar mucho a esto por todo lo que implica el movimiento, el juego.... Al final somos personas y la fuerza está en el grupo ¿qué más da si eres chico/chica, negro/blanco, rubia o morena? Creo que toda diferenciación ya desde que nacemos es negativa (GDM).

Los expertos y expertas manifiestan su acuerdo en que la aceptación, la tolerancia y el respeto deberían de ser las pautas comunes sobre las que se articule la EF. Afirman que el movimiento es el principal agente de aprendizaje desde las primeras etapas, destacando el rol del docente como fundamental:

Si queremos que los niños y niñas de hoy sean ciudadanos tolerantes y respetuosos en el mañana, es fundamental que las propuestas que planteemos en clase sean plenamente coeducativas [...] (EE6). Aceptar nuestras igualdades o diferencias, y más en EF, todavía sigue siendo uno de los principales retos a conseguir (EE4). Si todavía nos empeñamos en seguir calificando pruebas físicas en función de test estandarizados, estamos reproduciendo clara e implícitamente modelos de superioridad de género. Además, ¿qué aprendizaje conlleva esto? (EE1). La EF, bien enfocada, puede ser una herramienta de trabajo idónea para que sean comprendidas y bien entendidas desde el inicio las diferencias que existen entre chicos y chicas a nivel biológico y fisiológico [...]. A partir de esa normalización empezar a trabajar bajo parámetros de igualdad (EE7).

\section{Enfoques y modelos pedagógicos aplicados a la EF para conseguir tolerancia, igualdad y respeto en el aula (243 extractos de texto)}

Uno de los aspectos que más destaca el alumnado es la diferente visión que ha tenido en esta experiencia en relación a la que vivenció como estudiante. Manifiesta que independientemente del contenido que se trabaje lo importante es el cómo, y sobre todo poder aplicar un componente reflexivo para la igualdad a lo largo de las clases:

Me ha llamado muchísimo la atención ver cómo puede cambiar la EF dependiendo de la metodología que utilice el docente [...]. Hemos hecho contenidos totalmente diferentes y sobre todo colaborando unos con otros. Algunas cosas que hice en la ESO en $\mathrm{EF}$, y de las que no tuve buen recuerdo, las hemos vuelto a hacer aquí de un modo totalmente diferente [...]. Eso depende de cómo lo integre el profesorado. Siempre hemos reflexionado sobre lo que hacemos en clase, para qué lo hacemos y cómo la potencia del aprendizaje radica en el grupo, chicos y chicas [...]. Por muchas cosas que seas 
capaz de lograr si no lo hacen todos y todas no es lo mismo. El respeto y la coeducación verdaderamente surge cuando las actividades son interesantes, y no obligándoles a ponerse en parejas o grupos mixtos (GDM).

Los expertos y expertas consideran que la materia de por sí supone una verdadera oportunidad para poder trabajar la coeducación y género. Sin embargo, si no se aplican los enfoques metodológicos adecuados puede convertirse en todo lo contrario, siendo fuente de discriminación, intolerancia e intransigencia.

Ya sabemos que la EF tiene una gran cantidad de componentes para trabajar la coeducación e inclusión, pero también puede ser fuente de conflicto y desmotivación de los/as estudiantes hacia la asignatura (EE3). Si únicamente trabajamos desde la individualidad y la competición, es muy difícil que el día de mañana nuestros niños y niñas sean respetuosos y tolerantes y partan de la aceptación inicial en el trato directo e indirecto con personas (EE4). Si desde el principio no naturalizamos la situación desde el juego, la diversión y el aprendizaje, seguiremos reproduciendo modelos que vemos que no funcionan y que tiene graves consecuencias en algunos casos [...] Para niños fútbol y para niñas combas no es más que un componente sociocultural que indirectamente legitimizamos en EF (EE6). Los modelos pedagógicos (hay mucha literatura sobre esto) es la clave actual [...]. Tanto en la formación inicial como en la permanente del profesorado es donde deberíamos de dirigir nuestros esfuerzos (EE5).

\section{Discusıón}

Se ha observado cómo el enfoque relacional y crítico aplicado a la EF ha sido plenamente satisfactorio para los/as futuros/as maestros y maestras de cara a trabajar la coeducación y la igualdad de género en el futuro. Los expertos y expertas consideran la necesidad imperiosa de reorientar la EF hacia modelos que integren lo corporal y el movimiento como medio del fomento de las relaciones positivas entre iguales en lugar de aquellos más vinculados al rendimiento deportivo individual.

En relación a la primera categoría de análisis, los/as estudiantes perciben un cambio sustancial de la EF que vivenciaron en primaria y secundaria respecto a la presente experiencia. Este cambio radica, en parte, en el carácter social e inclusivo con la que se ha enfocado. Que futuros/as maestros y maestras en la etapa de primaria, siempre que se produzca un cambio metodológico, consideren la EF como una herramienta idónea para el trabajo de coeducación e igualdad de género, supone dos cosas: a) que los modelos participativos y de aprendizaje han de imponerse sobre los modelos tradicionales en la materia y b) que es preciso un cambio en las aulas de la educación obligatoria que verdaderamente conexione con la problemática social existente. Como afirman Anderson-Nathe, Gringeri y Wahab (2013), aquellos alumnos y alumnas que reciban una formación más centrada en los valores y las emociones que en la instrumentalización de los contenidos serán aquellos ciudadanos y ciudadanas que más reflexionen críticamente por los modelos imperantes socialmente aceptados. En este sentido, experiencias en EF han demostrado que tras la implantación de propuestas de actividad física en centros educativos con el lema "por y para todos/as" se mejoraron significativamente variables como la aceptación, la violencia injustificada y el clima motivacional en el 
aula (Hayden et al., 2014). El personal experto de la presente investigación parte de la premisa de que el movimiento es el principal agente de aprendizaje y socialización, pero que para ello el profesorado ha de tener claro que desde la EF no podemos seguir repitiendo planteamientos que quizás funcionaron hace décadas, ya que todo ha cambiado vertiginosamente. Es por ello que el trabajo coeducativo, de aceptación y de tolerancia se ha demostrado alcanzar en el momento en el que priman los objetivos de percepción de logro grupales sobre los resultados satisfactorios individuales y excluyentes (Kombe, Che, Carter y Bridges, 2016).

Respecto a la segunda categoría de análisis, los/las futuros maestros/as dan mucha importancia al rol que tiene el grupo a la hora de obtener experiencias satisfactorias. Admiten que haber trabajado el currículo oculto de manera implícita y real les ha dado una mayor consciencia sobre la discriminación existente en las clases y sobre la que no quieren incidir en su futuro profesional. En este sentido, diversas experiencias aplicadas en la FIP han demostrado que los/as estudiantes admiten haber adquirido más competencias profesionales cuando se han trabajado en las asignaturas ejemplos específicos sobre discriminación y género, así como determinadas estrategias para trabajarlo (Breneselovic y Krnjaja, 2016). Esta idea es complementada por el personal experto quien aconseja el tratamiento de temas como la evaluación, la normalización y la aceptación de las diferencias de una manera lógica. En esta línea, Mistry and Sood (2016) indican que el primer paso para que los niños y niñas asuman la igualdad en el trato, en derechos y en oportunidades en función del género es precisamente trabajar que hay cosas que nos diferencian. Así, en EF es importante que desde el principio ellos y ellas sepan que, a modo de ejemplo, biológicamente el hombre desarrolla unos niveles de fuerza superiores y por su parte, la mujer mejores niveles de flexibilidad. Esto no debe diferenciarnos, sino comprendernos mejora para alcanzar mejores niveles de complementación.

En relación a la tercera categoría del estudio, los estudiantes destacan dos aspectos: a) la metodología empleada por el docente de EF y b) el componente reflexivo que es necesario aplicar en el aula. Ya ha quedado demostrado que el rendimiento académico, entendido como la calificación obtenida en una asignatura, normalmente a partir de la memorización de contenidos, no tiene ninguna transferencia real en las competencias que demanda actualmente la sociedad actual (DeWall, 2016). Por ello, y más si cabe en la etapa de primaria, hay que trabajar propuestas que fomenten la creatividad, la autonomía, la responsabilidad y las competencias de trabajar en equipo. Esto es perfectamente abordable desde la EF siempre que se apliquen modelos pedagógicos abiertos y participativos en los que prime la aceptación propia y personal, el respeto al grupo independientemente de sus características y la integración de lo corporal en prácticas a través de retos grupales. Los expertos y expertas indican al respecto que si bien la EF puede convertirse en una herramienta que verdaderamente potencie la coeducación e igualdad de género, si no se utiliza la metodología adecuada puede tener el efecto contrario, generando discriminación, burlas, falta de autoestima y de autoconcepto. Determinadas evidencias científicas demuestran que debido a las malas experiencias obtenidas en la EF muchas personas no vuelven a realizar actividad física de manera regular como medio de socialización y divertimento (Landolfi, 2014). 


\section{CONCLUSIONES}

En este apartado se establecerá una conclusión para cada uno de los objetivos de la investigación, para posteriormente comentar el principal aporte del estudio, sus limitaciones y el interés que puede derivar para profesionales de este ámbito.

Respecto al primer objetivo, se concluye la relevancia que otorgan los/as futuros/ as maestros y maestras a la metodología y al enfoque aplicado en EF en la FIP para abordar la coeducación y la igualdad de género en el aula. Respecto al segundo objetivo, las personas expertas coinciden en destacar la necesidad de romper definitivamente el modelo tradicional de EF basado en el rendimiento deportivo individual, ya que de este modo se limita enormemente las posibilidades sociales y afectivas que dan esencia al área.

El principal aporte del estudio ha sido demostrar cómo la EF puede convertirse en una herramienta idónea para que ya desde las primeras etapas primen los aspectos de socialización, tolerancia, respeto y aceptación independientemente del género. Sin duda, y con un enfoque adecuado, esto puede repercutir a corto/medio plazo a obtener una normalización e igualdad de la que actualmente todavía se carece. Sin embargo, el estudio presenta algunas limitaciones. En primer lugar, únicamente se ha desarrollado una experiencia en una asignatura concreta en el ámbito universitario, por lo que se podrían llevar a cabo estudios de corte más longitudinal. En segundo lugar, solamente se da voz a futuros/as maestros/as y expertos/as en la temática. Sería de interés completar estos resultados analizando la percepción de las familias y de maestros/as ya ejercientes en la etapa de primaria y/o de secundaria. Consideramos que el artículo puede ser de especial utilidad para el profesorado de cualquier etapa educativa, interesados en implantar modelos pedagógicos en EF vinculados al trabajo de género y de coeducación desde una visión integradora y multidisciplinar. También para directores/as de centros educativos, coordinadores/ as académicos de planes de estudios en los Grados de Educación y responsables de la Administración Educativa, ya que podrán reflexionar profundamente sobre la relevancia que tiene apostar desde las primeras etapas por la elaboración de propuestas formativas que verdaderamente aborden el trabajo de la igualdad de género desde una perspectiva interdisciplinar y transversal. Se concluye destacando la idea de la importancia que tiene seguir investigando en un ámbito tan trascendente y del que queda tanto por avanzar.

\section{ReFERENCIAS BIBLIOGRÁFICAS}

Anderson-Nathe, B., Gringeri, C. y Wahab, S. (2013). Nurturing "Critical Hope" in Teaching Feminist Social Work Research. Journal of Social Work Education, 49(2), 277-291.

Bozkurt, E. y Tel, M. (2016). Opinions and Perceptions of Physical Education Students about Value Education. Educational Research and Reviews, 11(20), 1918-1924.

Breneselovic, D. P. y Krnjaja, Z. (2016). Discourses on Gender in Early Childhood Education and Care (ECEC) Setting: Equally Discriminated Against. Journal of Pedagogy, 7(2), 51-77. http://doi.org/10.1515/jped-2016-0011. 
Dervent, F. (2015). The Effect of Reflective Thinking on the Teaching Practices of Preservice Physical Education Teachers. Issues in Educational Research, 25(3), 260-275.

Chick, K. A. (2014). The Making of a Man: Rethinking and Challenging Stereotypes. Kappa Delta Pi Record, 50(4), 175-180. http://doi.org/10.1080/00228958.2014.9 60341.

Choi, K. M. y Protivnak, J. J. (2016). Leaping into the Unknown: Experience of Counseling Students Participating in Group Work with International Students. Journal for Specialists in Group Work, 41(3), 238-261. http://doi.org/10.1080/01933922. 2016.1197991.

Costigan, L. (2009). Assessing educational effectiveness: The focus group interview as a technique for data collection. Communication Education, 39(2), 117-127. http:// doi.org/10.1080/03634529009378794.

DeWall, N. (2016). Millennials by Heart: Memorization as an Active Learning Strategy for the "SparkNotes" Generation. Journal on Excellence in College Teaching, 27(4), 77-91.

Finco, M. D. et al. (2015). Exergaming as an Alternative for Students Unmotivated to Participate in Regular Physical Education Classes. International Journal of GameBased Learning, 5(3), 10-15. http://doi.org/10.4018/IJGBL.2015070101.

Hayden, L. et al. (2014). Integrating Physical Activity, Coach Collaboration, and Life Skill Development in Youth: School Counselors' Perceptions. Journal of School Counseling, 12(23), 38-44.

Hortigüela, D., Fernández-Río, J. y Pérez-Pueyo, A. (2016a). Efectos del planteamiento docente en la enseñanza del fútbol sobre el clima de aula. Percepciones de alumnado y profesorado. Cuadernos de Psicología del Deporte, 16(1), 295-306.

Hortigüela, D., Fernández-Río, J. y Pérez-Pueyo, A. (2016b). Long-term effects of the pedagogical approach on the perceptions of physical education by students and teachers. Journal of Physical Education and Sport, 16(4), 1326-1333. http://doi. org/10.7752/jpes.2016.04210.

Khasnabis, D. y Upton, K. (2013). Disrupting Traditions: Swimming against the Current of Adolescent Bullying. Voices from the Middle, 20(3), 37-46.

Kingdon, D., Serbin, L. A. y Stack, D. M. (2017). Understanding the Gender Gap in School Performance among Low-Income Children: A Developmental Trajectory Analysis. International Journal of Behavioral Development, 41(2), 265-274. http:// doi.org/10.1177/0165025416631836.

Kombe, D., Che, S., Carter, C. y Bridges, L. (2016). Student Academic Self-Concept and Perception of Classroom Environment in Single-Sex and Coeducational Middle Grades Mathematics Classes. School Science and Mathematics, 116(5), 265-275.

Landolfi, E. (2014). Teachers' Understanding of Students' Attitudes and Values toward Physical Activity in Physical Education Dropout Rates and Adolescent Obesity. Physical Educator, 71(3), 12-23.

Mills, M., McGregor, G., Baroutsis, A., Te Riele, K. y Hayes, D. (2016). Alternative Education and Social Justice: Considering Issues of Affective and Contributive Justice. Critical Studies in Education, 57(1), 100-115. http://doi.org/10.1080/175084 87.2016.1087413. 
Mistry, M. y Sood, K. (2016). Busting the Myth of Gender Bias: Views from Men and Women Primary-School Trainees and Teachers. Education, 3(13), 283-296. http:// doi.org/10.1080/03004279.2014.922595.

Parker, A., Bush, A. y Yendol-Hoppey, D. (2016). Understanding Teacher Candidates' Engagement with Inquiry-Based Professional Development: A Continuum of Responses and Needs. New Educator, 12(3), 221-242. http://doi.org/10.1080/15476 88X.2015.1027978.

Pérez-Pueyo, A., Hortigüela, D. y Hernando, A. (2014). La coevaluación intragrupal y el reparto de notas bajo un proceso de evaluación formativa. En P. Membiela, N.

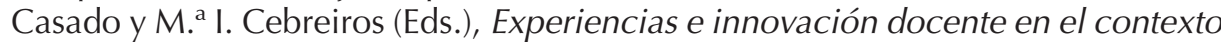
actual de la docencia universitaria (pp. 285-289). Ourense: Educación Editora.

Romano, N. C., Donovan, C., Chen, H. y Nunamaker, J. F. (2014). A Methodology for Analyzing Web-Based Qualitative Data. Journal of Management Information Systems, 19(4), 213-246.

Rosen, L. H., Scott, R. y DeOrnellas, K. (2017). Teachers' Perceptions of Bullying: A Focus Group Approach. Journal of School Violence, 16(1), 119-139. http://doi.org /10.1080/15388220.2015.1124340.

Saldaña, J. (2009). The coding manual for qualitative researchers. Singapore: Sage.

Strauss, A. y Corbin, J. (2002). Bases de la investigación cualitativa: Técnicas y procedimientos para desarrollar la teoría fundamenta-da. Colombia: Universidad de Antioquia.

Temel, C. y Güllü, M. (2016). Social Gender in the Pictures Drawn by Students about Physical Education Class. Educational Research and Reviews, 11(8), 530-536.

Thorleif, L. (2007). The Qualitative-Quantitative Distinction: Some comments. Journal Scandinavian Journal of Educational Research, 49(2), 115-132. http://doi. org/10.1080/00313830500048790.

Varga-Atkins, T., Mclsaac, J. y Willis, I. (2015). Focus Group meets Nominal Group Technique: an effective combination for student evaluation? Innovations in Education and Teaching International, 1(3), 1-12. http://doi.org/10.1080/14703297.2 015.1058721. 